\title{
IMPORTÂNCIA DA INVASÃO NEURAL E LINFÁTICA NO PROGNÓSTICO DO ADENOCARCINOMA COLORRETAL
}

\author{
Antonio Paulo Durante, Sansom Henrique Bromberg*, Elci Barreto, \\ Giovanni Cappellano, Antonio Claudio de Godoy \\ Trabalho realizado na Pós-graduação em Gastroenterologia Cirúrgica do Instituto de Assistência \\ Médica ao Servidor Público Estadual (IAMSPE) e no Serviço de Gastroenterologia Cirúrgica \\ do Hospital do Servidor Público Estadual de São Paulo - \\ Francisco Morato de Oliveira (HSPE-FMO), São Paulo, SP.
}

RESUMO - OBjetivos. A evolução paradoxal de um terço dos doentes com neoplasias colorretais catalogadas no estádio B e C de Dukes mostra ser desejável a adição de outras variáveis prognósticas. 0 principal objetivo deste trabalho foi o de estudar 0 papel prognóstico da invasão linfática e neural em uma série de doentes submetidos à cirurgia curativa e acompanhados por longo período.

Métodos. Foram estudados 320 doentes com câncer colorretal submetidos à extirpação curativa, com idade mediana de 58 anos, sendo $199(62,8 \%)$ do sexo feminino. A invasão neural foi caracterizada pela presença de células cancerosas, infiltrando o perineuro e/ou o fascículo neural. A linfática, pela presença de células neoplásicas no interior de espaço limitado por endotélio, desprovido de fibras musculares e elásticas. Essas variáveis foram associadas à classificação original de Dukes.

Resultados. A invasão neural foi observada em $15 \%$ das peças extirpadas e a linfática em $\mathrm{I} 4,1 \%$. Os índices de invasão cresceram do ceco ao reto, local preferencial das mesmas. A sobrevida de cinco anos dos portadores de neoplasias com invasão neural foi de $25 \%$ em oposição a $64 \%$ daqueles sem invasão $(p<0,01)$. Para os com invasão linfática, a sobrevida foi de $26,7 \%$ e $63,3 \%$, respectivamente $(p<0,01)$. Independentemente do comprometimento ou não dos linfonodos, a sobrevida foi sempre pior na presença da invasão neural. Em portadores de linfonodos livres, a invasão linfática identificou subgrupo de doentes com pior prognóstico. A presença destas variáveis identificou nos portadores de tumores Dukes B, subgrupo de pior prognóstico.

Conclusãa. A presença de invasão neural e linfática no adenocarcinoma colorretal está associada a prognóstico desfavorável de seus portadores.

Unitermos: Neoplasias colorretais. Metástase linfática. Invasão neural. Prognóstico.

\section{INTRODUÇÃo}

O prognóstico dos portadores de carcinoma colorretal é dependente da extensão anatômica do tumor por ocasião do diagnóstico e da possibilidade de se realizar a extirpação completa da neoplasia. Satisfeitas estas exigências, passam a influenciar o prognóstico outras variáveis, entre as quais se destaca o estudo histopatológico.

Após cerca de sete décadas, a classificação de Dukes' permanece sendo o método mais utilizado no estadiamento do carcinoma colorretal, predizendo o prognóstico em grande número de casos. No entanto, pacientes com tumores na mesma classe de Dukes apresentam muitas vezes evoluções paradoxais ${ }^{2,3}$.

* Correspondência:

Av. Angélica, 589 - Apto. 81 - Cep: 01227-000 São Paulo - SP - Tel.: (11) 3826.2600

E-mail: apsped@terra.com.br - mbrombe@attglabal.net
Torna-se necessário adicionar outras variáveis morfológicas que possibilitem estadiamento mais completo e, por conseguinte, avaliem com melhor precisão o prognóstico desses doentes ${ }^{3-6}$.

No estudo convencional, parâmetros como a invasão linfática e a invasão neural pela neoplasia ainda não ganharam amplo reconhecimento de seus méritos.

Em vários trabalhos a invasão dos vasos linfáticos não influenciou o prognóstico de doentes com neoplasias colorretais $5,7,8$. No entanto, também foi constatada significante redução da sobrevida em portadores de lesões exibindo células tumorais nos vasos linfáticos ${ }^{9-15}$.

Mais recentemente, Compton et al., analisando múltiplos fatores prognósticos destas neoplasias, incluíram a invasão dos vasos linfáticos como fator de grande importância prognóstica ${ }^{6}$. O mesmo se constata em relação à invasão neural. Sua presença foi associa- da com maior recidiva local e reduzida sobrevivência ${ }^{5,16-18}$. Outros autores concluíram ser 0 valor prognóstico da invasão neural insuficientemente estudado ou sem influência prognóstica, ${ }^{6,19}$

O emprego dessas duas variáveis poderia auxiliar na identificação de subgrupos de pacientes com neoplasias catalogadas na mesma classe de Dukes. A literatura nacional carece de estudos a respeito ${ }^{4}$. Por essas razões, decidimos estudar o papel desempenhado pela invasão linfática e neural, e relacioná-las com a classificação de Dukes, em uma série de doentes com carcinoma colorretal submetidos a procedimento cirúrgico curativo e acompanhados por longo período.

\section{Métodos}

Foram estudados 320 portadores de adenocarcinoma colorretal submetidos à extirpação cirúrgica curativa no Serviço de 
DURANTE AP ET AL.

Gastroenterologia Cirúrgica do Hospital do Servidor Público Estadual de São Paulo Francisco Morato de Oliveira (HSPE-FMO), entre 1964 e 1980.

A média de idade foi de $58,1 \pm 12$ anos, variando de 28 a 86 anos e a mediana de 58,0 anos. Havia 199 doentes do sexo feminino $(62,8 \%)$ e 121 do sexo masculino $(37,2 \%)$.

Os doentes foram seguidos até o óbito, sempre motivado pela neoplasia em estudo, ou no mínimo por cinco anos. O período menor de acompanhamento foi de três meses e o maior de 295 meses, com média de 80,8 $\pm 63,9$ meses

Oitenta e uma das neoplasias $(25,3 \%)$ estavam assestadas no colo proximal, 104 no distal (32,5\%) e 135 no reto (42,2\%).

Foram efetuados os seguintes procedimentos operatórios: 64 colectomias direitas, 87 colectomias esquerdas, 14 sigmoidectomias, 96 amputações abdomino-perineais, 21 reto-colectomias abdominais, 15 retocolectomias abdomino-perineais, 16 transversectomias, quatro colectomias totais e três procto-colectomias totais.

As cirurgias foram realizadas por pequeno grupo de cirurgiões com a mesma formação, empregando idêntica padronização. Em todos os casos a equipe cirúrgica considerou como curativa a extirpação da neoplasia. Entendeu-se como cirurgia curativa aquela onde a equipe concluiu pela ausência de crescimento neoplásico à distância do tumor primitivo e inexistência de neoplasia residual visível, associada ao não comprometimento das margens da peça extirpada constatada pelo patologista.

O estudo anatomopatológico foi realizado por patologista do mesmo hospital, que revisou todas as lâminas estocadas, desconhecendo a evolução clínica dos doentes. Sempre que necessário os blocos de parafina foram reprocessados para estudo com hematoxilina e eosina.

A invasão neural foi caracterizada como positiva quando as células cancerosas infiltravam o perineuro ou situavam-se no interior do fascículo neural (Figura I).

A invasão dos vasos linfáticos foi definida pela presença de células neoplásicas no interior de um espaço limitado por endotélio, desprovido de fibras musculares lisas murais, fibras elásticas e células sanguíneas vermelhas (Figura 2).

\section{Figura I - Invasão neural. Células neoplásicas no interior do fascículo neural (Hematoxilina-Eosina; I00X)}

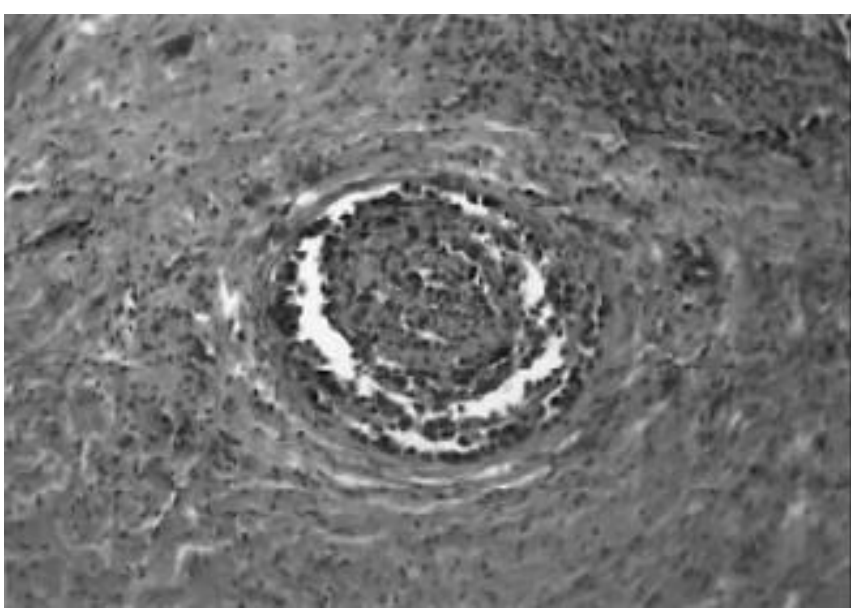

Figura 2 - Invasão dos vasos linfáticos. Células neoplásicas no interior de um espaço limitado por endotélio(Hematoxilina eEosina; 100X)

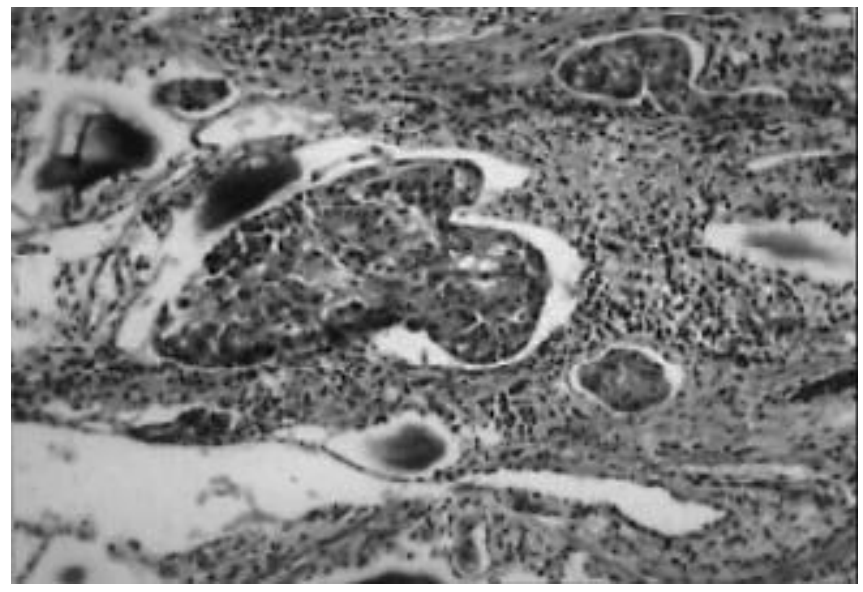

Todos os espécimes cirúrgicos foram estagiados de acordo com a classificação original de Dukes'. Considerou-se como Dukes $A$ as neoplasias restritas à parede do segmento intestinal, e como Dukes B as que se estendiam por toda a parede, atingindo inclusive o tecido adiposo adventício. Como C, as com linfonodos comprometidos. Em nove das peças cirúrgicas não foram encontrados nodos linfáticos. Deste modo a classificação de Dukes empregada neste trabalho pode ser observada na Tabela I.
Para análise estatística utilizou-se nível de significância de 5\% $(a=0,05)$. Para Tabelas $2 \times 2$, utilizou-se o teste do Qui-quadrado corrigido segundo Yates. Quando presentes mais de duas variáveis (tabelas $2 \times \mathrm{N}$ ) efetuou-se o teste do Qui-quadrado comum. $\mathrm{O}$ teste de partição do Qui-quadrado para tabelas $2 \times \mathrm{N}$ foi utilizado na presença de mais de duas modalidades. Nessas tabelas, os grupos foram identificados pelas letras maiúsculas do alfabeto, entre parênteses (A), (B), (C), etc. Quando das restrições numéricas, foi utilizado o teste exato de Fisher. 


\begin{tabular}{ccc}
\hline \multicolumn{3}{c}{ Tabela I - Distribuição de 320 neoplasias colorretais, segundo a classificação de Dukes } \\
\hline Classe & N & $\%$ \\
A & 44 & 13,7 \\
B & 156 & 48,8 \\
C & 120 & 37,4 \\
\hline
\end{tabular}

Tabela 2 - Distribuição da invasão neural e linfática, segundo o local do intestino

\begin{tabular}{|c|c|c|c|c|c|}
\hline LOCAL & $n$ & IN & $\%$ & IL & $\%$ \\
\hline Colo proximal & 81 & 6 & 7,4 & 9 & $|1|$, \\
\hline Colo distal & 104 & 14 & 13,5 & 12 & 11,5 \\
\hline Reto & 135 & 28 & 20,7 & 24 & 17,8 \\
\hline TOTAL & 320 & 48 & 15,0 & 45 & 14,1 \\
\hline
\end{tabular}

IN: invasão neural

IL: invasão linfática

\begin{tabular}{|c|c|c|c|}
\hline \multicolumn{4}{|c|}{$\begin{array}{l}\text { Tabela } 3 \text { - Sobrevida de cinco anos de } 320 \text { doentes com carcinomas colorretais, segundo a } \\
\text { presença de invasão neural e linfática }\end{array}$} \\
\hline \multirow{3}{*}{ presente } & $N(320)$ & Sobrevida $\geq 5$ anos & Valor de $p$ \\
\hline & 48 & $25,0 \%$ & \multirow{4}{*}{0,001} \\
\hline & & & \\
\hline ausente & 272 & $64,0 \%$ & \\
\hline \multirow[t]{2}{*}{ presente } & 45 & $26,7 \%$ & \\
\hline & & & \multirow[t]{2}{*}{0,001} \\
\hline ausente & 275 & $63,3 \%$ & \\
\hline \multirow[t]{2}{*}{ IN pres / IL pres } & 23 & $21,7 \%$ & \multirow{3}{*}{0,001} \\
\hline & & & \\
\hline IN aus / IL aus & 250 & $66,8 \%$ & \\
\hline$\overline{\mathrm{N}}$ - Invasão Neural & & & \\
\hline IL - Invasão Linfática & & & \\
\hline
\end{tabular}

Tabela 4 - Sobrevida de 3 I I doentes com carcinoma colorretal, segundo a associação entre grau de comprometimento doslinfonodos e invasãoneural

\section{Linfonodo/}

Invasãoneura

(A) livre / presente

(B) livre/ausente

(C) compr / presente

(D) compr/ausente

TOTAL

Compr = comprometido

$A \times B-p=0,003$ (Fisher)*

$C \times D-p=0,020$ (Fisher)*

$A \times C-p=0,289$ (Fisher)*

$B \times D-\chi^{2}=26,48 *$

$p<0,0001$
Sobrevida

\begin{tabular}{ccc}
$<\mathbf{5 a}$ & & $>\mathbf{5 a}$ \\
$\mathbf{N}$ & $\%$ & $\mathbf{N}$ \\
\hline 10 & 62,5 & 6 \\
44 & 25,1 & 131 \\
25 & 80,6 & 6 \\
51 & 57,3 & 38
\end{tabular}

130

$\chi^{2}=50,79 *$

181

$p<0,0001$

\begin{tabular}{c}
$\%$ \\
\hline 37,5 \\
74,9 \\
19,4 \\
42,7
\end{tabular}

42,7
Total

16

175

31

89

\section{Resultados}

A invasão neural esteve presente em 15\% (48/320) das peças cirúrgicas examinadas e a invasão dos vasos linfáticos em 14,1\% (45/ 320), como pode ser observado na Tabela 2.
Os índices de invasão neural e linfática cresceram do ceco ao reto, local preferencial das mesmas (Tabela 2).

No colo proximal, o comprometimento neural foi observado em seis das peças extirpa- das, predominando no ceco $(83,3 \%)$, seguido pela flexura direita (16,7\%). Já no colo distal ela foi constatada em 14 espécimes, e o predomínio se deu no sigmóide $(85,7 \%)$, seguido do descendente (14,3\%). A invasão linfática proximal foi observada em nove dos espécimes e foi mais encontradiça no ceco $(88,9 \%)$ e flexura direita $(1|| \%$,$) . No colo distal foi$ constatada em 12 das peças, predominando no sigmóide (83,4\%), seguida da flexura esquerda e descendente, ambos com incidência de 8,3\%.

A sobrevida de cinco anos de portadores de neoplasia colorretal que integraram este trabalho, sem levar em consideração nenhum parâmetro específico, foi de 58,1\%. Na presença das variáveis em estudo, associadas ou isoladamente, a sobrevida foi sempre significantemente menor (Tabela 3).

A sobrevida de cinco anos dos 191 doentes $(61,4 \%)$ com linfonodos livres foi de 71,7\%. Nos 120 portadores de linfonodos comprometidos $(38,6 \%)$ ela foi significantemente menor - 36,7\% ( $p<0,001)$. A sobrevida dos portadores de linfonodos livres e ausência de invasão neural foi significantemente maior que daqueles com linfonodos livres associados à presença dessa invasão $74,9 \% \times 37,5 \%$, respectivamente $(p=0,003)$. 0 mesmo foi observado em portadores de linfonodos comprometidos; quando da ausência de invasão neural ela foi de 42,7\%, caindo para 18,4\% na presença da mesma $(p=0,020)$. Esses dados podem ser observados com maiores detalhes na Tabela 4.

Da mesma maneira, em portadores de linfonodos livres, a presença de invasão linfática diminuiu de modo significante a sobrevida de cinco anos de seus portadores (Tabela 5).

A presença de invasão neural reduziu significantemente a sobrevivência de cinco anos dos doentes com tumores classificados na classe B e C de Dukes (Tabela 6).

De modo semelhante, a permeação linfática influenciou a sobrevida de cinco anos dos portadores de lesões catalogadas na classe B de Dukes (Tabela 7).

\section{Discussão}

O estudo da invasão neural no tubo digestório, preferencialmente, vem sendo realizado em lesões distais do intestino, interessando ao retosigmóide e reto, provavelmente devido à maior possibilidade de recidiva 


\begin{tabular}{lccccc}
\hline \multicolumn{6}{c}{ Tabela 5 - Sobrevida de 3II doentes com carcinoma colorretal, segundo a associação entre grau de } \\
comprometimento dos linfonodos e invasão dos vasos linfáticos
\end{tabular}

\begin{tabular}{|c|c|c|c|c|c|}
\hline \multicolumn{6}{|c|}{$\begin{array}{l}\text { Tabela 6 - Sobrevida de cinco anos de } 320 \text { doentes com câncer colorretal, segundo a } \\
\text { classificação de Dukes e invasão neural }\end{array}$} \\
\hline Classe/ & & jobrevi & & & \\
\hline Invasão neural & $\begin{array}{c}<5 a \\
N\end{array}$ & $\%$ & $\begin{array}{c}>5 \mathrm{a} \\
\mathrm{N}\end{array}$ & $\%$ & Total \\
\hline $\begin{array}{l}\text { (A) A / presente } \\
\text { (B)A/ausente } \\
\text { (C) B/presente } \\
\text { (D) B/ausente } \\
\text { (E) C/ presente } \\
\text { (F)C/ausente } \\
\text { TOTAL }\end{array}$ & $\begin{array}{c}1 \\
3 \\
10 \\
44 \\
24 \\
52 \\
134\end{array}$ & $\begin{array}{l}50,0 \\
7,1 \\
66,7 \\
31,2 \\
80,0 \\
57,8\end{array}$ & $\begin{array}{c}1 \\
39 \\
5 \\
97 \\
6 \\
38 \\
186\end{array}$ & $\begin{array}{l}50,0 \\
92,9 \\
33,3 \\
68,8 \\
20,0 \\
42,2\end{array}$ & $\begin{array}{c}2 \\
42 \\
15 \\
141 \\
30 \\
90 \\
320\end{array}$ \\
\hline
\end{tabular}

Os índices deste trabalho situam-se na média dos relatados, sendo de $15 \%$ para invasão neural e de 14,1\% para a linfática.

O número de trabalhos versando exclusivamente sobre invasão linfática é reduzido, pois geralmente ela é estudada em conjunto com a invasão venosa. Na realidade, constatase grande variabilidade entre os observadores no reconhecimento de pequenos vasos, principalmente na diferenciação entre linfáticos e vênulas pós-capilares. Quer a presença de artefatos ocasionados pela fixação tecidual quer a destruição da arquitetura vascular pela neoplasia contribuam para essa variabilidade no reconhecimento daquelas estruturas ${ }^{6}$.

O papel prognóstico da invasão neural remonta a meados do século XIX ${ }^{23}$, havendo estudos detalhados em muitos órgãos digestivos $^{23-26}$. No primeiro trabalho realizado sua presença associou-se a significante aumento da recidiva local $(81,2 \%)^{18}$. Pesquisas posteriores vieram comprovar estes achados $^{9,17,20,21}$. Lasser et al. ${ }^{27}$, em 400 casos de câncer retal, observaram redução na sobrevivência de cinco anos de $62 \%$ para $28 \%$ quando da presença de invasão neural. Essas porcentagens são muito próximas das obtidas neste estudo, onde a presença da invasão neural reduziu a sobrevida de seus portadores de $64 \%$ para $25 \%$.

A invasão perineural indicaria o potencial da neoplasia crescer localmente; células tumorais foram encontradas além de $10 \mathrm{~cm}$ do tumor primário ao longo dos espaços perineurais $^{16,18}$.

A invasão neural foi considerada como parâmetro de não-radicalidade cirúrgica e portanto, variável adversa ao melhor prognóstico; com freqüência mostrou-se associada ao crescimento infiltrativo das bordas neoplásicas ${ }^{6}$. Também foi correlacionada à presença de metástases hepáticas ${ }^{21}$ e sua associação com a recidiva local seria indicadora de crescimento mais agressivo da neoplasia ${ }^{20}$.

No entanto, a associação entre invasão neural e menores índices de sobrevida não é consenso da literatura. Estudo de 82 pacientes com câncer colorretal concluiu que o envolvimento perineural das peças extirpadas não afetou o prognóstico ${ }^{19}$. A conclusões semelhantes chegaram outros autores ${ }^{4,6}$.

A presença de invasão neural em portadores de linfonodos livres identificou neste estudo subgrupo com menor sobrevida de local quando da sua presença ${ }^{20,21}$. Seefeld e Bargen ${ }^{18}$ foram os primeiros a estudar a presença da invasão neural no carcinoma retal, encontrando-a em 30\% dos espécimes estudados. Sua incidência nas peças extirpadas tem variado de $9,9 \%$ a $34,5 \%{ }^{17,20,21}$. No Brasil, Rawet constatou sua existência em $31 \%$ dos 84 espécimes estudados ${ }^{4}$. Já os índices de comprometimento vascular linfático variaram de $12,5 \%$ a $40,9 \% 11,22$. 
cinco anos quando comparados aos com ausência desta invasão - 37,5\% x 74,9\%, respectivamente (Tabela 5). Assim, em nossa opinião, doentes com tais características deveriam ser submetidos a tratamento quimioterápico complementar e intensivo acompanhamento.

O valor prognóstico da invasão dos vasos linfáticos também é controverso, predominando os que realçam sua importância prognóstica? ${ }^{9} 12-15$, embora outros estudiosos não tenham constatado tal influência ${ }^{5,7,19}$. Ela não foi considerada de importância prognóstica quando do estudo de 211 carcinomas retais no estádio $\left.\right|^{8}$. Já Korenaga et al. ${ }^{28}$ observaram em 113 portadores de carcinoma colorretal, significante redução na sobrevivência de cinco anos - de $74,5 \%$ para $39,6 \%$, quando da sua presença. Outro estudo mostrou que em espécimes exibindo embolização linfática, a sobrevivência de cinco anos caiu assustadoramente, de $71 \%$ para $3 \%{ }^{14}$. Em pólipos cancerizados extirpados por endoscopia, a presença desta variável associou-se ao aparecimento de metástases hepáticas e nos linfonodos ${ }^{29}$.

A demonstração de invasão linfática em portadores de linfonodos negativos faria crescer sua importância, pois identificaria enfermos com risco aumentado de desenvolver metástases ${ }^{20}$.

Tal fato foi constatado no presente estudo. Os portadores de neoplasias sem comprometimento dos linfonodos e sem invasão linfática apresentaram sobrevida de cinco anos de $75,4 \%$, significantemente maior que a de $31,2 \%$ na presença de invasão. Conseguiu-se, pois, identificar subgrupo de doentes com prognóstico desfavorável, representado pela ausência de comprometimento dos linfonodos e com invasão dos vasos linfáticos provavelmente capaz de se beneficiar de terapêutica complementar.

Os achados deste trabalho estão de acordo com aqueles que demonstraram a influência negativa desse parâmetro na sobrevida, pois quando presente a sobrevivência diminuiu de $63,3 \%$ para $26,7 \%$ (Tabela 3).

Poucos trabalhos avaliaram o papel prognóstico da presença simultânea da invasão neural e linfática9,16. A presente pesquisa constatou expressiva sobrevivência dos doentes quando da ausência concomitante da invasão neural e linfática (66,8\% - Tabela 3). Quando aquelas variáveis estavam presentes, associadas ou isoladamente, a sobrevivência foi sempre significantemente menor. Estudos utilizando corantes ou microscopia eletrônica mostraram a presença de comunicações entre o perineuro e capilares linfáticos, fato capaz de explicar a associação entre invasão neural e a linfática ${ }^{16,18,21,26}$. No entanto, esta opinião não é compartilhada por outros estudiosos ${ }^{30}$.

A presença dessas duas variáveis em neoplasias estagiadas na classe B de Dukes identificou doentes de pior prognóstico, quando comparados aos sem a presença das mesmas (Tabelas 6 e 7). Estes dados justificam a inclusão rotineira dessas duas variáveis nos relatos anatomopatológicos.

De fundamental importância neste estudo foi a constatação de que a presença de invasão linfática e neural associaram-se a menores índices de sobrevivência. A nosso ver, esses parâmetros influenciam a história natural da doença. Portadores destas lesões, independentemente das classificações utilizadas, deveriam ser selecionados para terapêutica complementar e seguimento intensivo.

\section{SUMMARY}

\section{Prognostic value of lymphatic VESSEL AND NEURAL INVASION IN COLORECTAL CARCINOMA}

ObjetINES. The paradoxical evolution of approximately one third of patients with coloretal cancer cataloged in Dukes stages B and C demonstrates the necessity of make useful other prognostic criteria. The presence of neural and lymphatic invasion of tumor cells was correlated with the prognosis of patients submitted to radical operation.

Methods. We performed a retrospective study on 320 patients with colo-rectal carcino$\mathrm{ma}$, with mean age 58 years and 199 (62.2\%) females. Neural invasion was accessed as positive if cancer cells infiltration into the perineurium or neural fasciculus was detected at the leading point. Lymphatic invasion was defined by cancer cells infiltration into a space limited by endothelium without muscular or elastic fibers. Those variable were associated to the original classification of Dukes.

RESULTS. Lymphatic and neural invasion was demonstrated in $14.1 \%$ and $15 \%$ of 320 operation specimens respectively. The most frequent site of lymphatic and neural invasion was the rectum. The overall sunvival was $25 \%$ in the presence of neural invasion and $64 \%$ without neural invasion $(p<0.01)$. At the presence of lymphatic invasion, the overall survivals were $26.7 \%$ and $63.3 \%$, respectively $(p<0.01)$. The overall survival was always worse in the presence of the invasion neural, indenpendently of the compromising or not of the lynphonodes. In patients of free lynphonodes, the lymphatic invasion identifird sub-groups of sick with worse prognosis. The presence of these variable it identified in patients with tumors Dukes B, subgroup of worse prognosis.

CONCLUSION. Neural and lymphatic invasion are important ways of spread of colorectal cancer and the presence of both is associated with worse prognosis. [Rev Assoc Med Bras 2004; 50(I): 21-6]

KEY WORDS: Colorectal cancer. Neural invasion. Lymphatic invasion. Prognosis. Lymphatic metastasis

\section{REFERÊNCIAS}

I. Dukes CE. The classification of cancer of the rectum. J Pathol Bacteriol 1932; 35:323-32.

2. Chapuis PH, Dent OF, Fisher R, Newland RC, Chapuis $\mathrm{PH}$, Bokey EL, et al. A multivariate analysis of clinical and pathological variables in prognosis after resection of large bowel cancer. Br J Surg 1985; 72:698-702.

3. Bromberg, SH, Barreto E, Moncau JEC, Goldenberg S. Estudo de fatores morfológicos prognósticos do câncer colo-retal extirpado. Acta Cir Bras 1997; 12:206-12.

4. Rawet V. Carcinoma colorretal: estadiamento e parâmetros prognósticos [tese]. São Paulo: Faculdade de Medicina, Universidade de São Paulo; 1998.

5. Mulcahy HE, Toner M, Patchett SE, Daly L, O' Donoghue DP. Identifying stage B colorectal cancer patients at high risk of tumor recurrence and death. Dis Colon Rectum 1997; 40:326-31.

6. Compton CC, Fenoglio-Preiser CM, Pettigrew N, Fielding LP. American Joint Committee on Cancer Prognostic Factors Consensus Conference: Colorectal Working Group. Cancer 2000; 88:1739-57.

7. Khankhanian N, Mavligit GM, Russell WO, Schimek M. Prognostic significance of vascular invasion in colorectal cancer of Duker' B class. Cancer 1977; 39:1 195-200.

8. Blumberg D, Paty PB, Picon Al, Guillem JG, Klimstra DS, Minsky DB, et al. Stage I rectal cancer: identification of high-risk patients. J Am Coll Surg 1998; 186:574-80.

9. SprattJS, Spjut HJ. Prevalence and prognosis of individual clinical and pathological variables associated with colorectal carcinoma. Cancer 1967; 20: 1976-85.

10. Brandão O, Sobrinho-Simões MA, Serrão D. Prognosis in colorectal carcinoma: a reassessment of the pathologist's role. Pathol Res Pract 1985; 180:501-10. 
II. De Leon ML, Schoetz DJ Jr, Coller JA, Veidenheimer MC. Colorectal cancer: Lahey Clinic experience, 1972-1976: an analysis of prognostic indicators. Dis Colon Rectum 1987; 30:237-42.

12. Michelassi F, Block GE, Vannucci L, Montag A, Chappell R. A 5- to 21-years follow-up and analysis of 250 patients with rectal adenocarcinoma. Ann Surg 1988; 208:379-89.

13. Minsky BD, Mies C, Rich TA, RechtA. Lymphatic vessel invasion is an independent prognostic factor for survival in colorectal cancer. Int | Radiat Oncol Biol Phys 1989; 17:31 I-8.

14. Garcia Peche P, Vazquez Prado A, Fabra Ramis R, Trullenque Peris R. Factors of prognostic value in Long-term survival of colorectal cancer patients. Hepatogastroenterol | 991; 38:438-43

15. Shirouzu K, Isomoto H, Morodomi T, Kakegawa T. Carcinomatous lymphatic permeation. Prognostic significance in patients with rectal carcinoma - a long term prospective study. Cancer 1995; 75:4-10.

16. Knudsen JB, Nilsson T, Sprechler M, Johansen A, Christensen N. Venous and nerve invasion as prognostic factors in postoperative survival of patients with resectable cancer of the rectum. Dis Colon Rectum 1983: 26:6। 3-7.

17. Bognel C, Rekacewicz C, Mankarios H, Pignon JP, Elias D, Duvillard P, et al. Prognostic value of neural invasion in rectal carcinoma: a multivariate analysis on 339 patients with curative resection. Eur J Cancer 1995; 31:894-8.
18. Seefeld PH, Bargen JA. The spread of carcinoma of the rectum, invasion of lymphatics, veins and nerves. Ann Surg 1943; I | 8:76-90.

19. De Mascarel A, Coindre JM, De Mascarel I, Trojani M, Maree D, Hoerni B. The prognostic significance of specific histologic features of carcinoma of the colon and rectum. Surg Gynecol Obstet 1981; 153:5 I I-4.

20. Horn A, Dahl O, Morild I. Venous and neural invasion as predictors of recurrence in rectal adenocarcinoma. Dis Colon Rectum 1991; 34:798-804.

21. Shirouzu K, Isomoto H, Kakegawa $T$. Prognostic evaluation of perineural invasion in rectal cancer. Am J Surg 1993; 165:233-7.

22. Hermanek P, Guggenmoos-Holzmannn I, Gall FP. Prognostic factors in rectal carcinoma. A contribuition to the further development of tumor classification. Dis Colon Rectum 1989; 32:593-9

23. Ballantyne AJ, Mccarten AB, Ibanez MI. The extension of cancer of the head and neck through peripheral nerves. Am | Surg 1963; 106:65I-67.

24. Ozaki H, Hiraoka T, Mizumoto R, Matsuno $S$, Matsumoto $Y$. The prognostic significance of lymph node metastasis and intrapancreatic perineural invasion in pancreatic cancer after curative resection. Surg Today 1999; 29:16-22.

25. Kayahara M, Nagakawa T, Tsukioka Y, Ohta T, Ueno K, Miyazaki I. Neural invasion and nodal involvement in distal bile duct cancer Hepatogastroenterol 1994; 41:190-4.

26. Tanaka A, Yoshikawa H, Okuno K, Koh K, Machidera K, Kubo K, et al. The importance of neural invasion $(\mathrm{NI})$ as a prognostic factor in diffuse invasive gastric cancer. Surg Today 1997; 27:692-5.

27. Lasser P, Mankarios H, Elias D, Bognel C. Eschwege $F$, Wibault $P$, et al. Etude pronostique uni et multi-factorielle de 400 adénocarcinomes rectaux réséqués. J Chir 1993; 130:57-65.

28. Korenaga D, Ueo H, Mochida K, Kusumoto T, Baba H, Tamura S, et al. Prognostic factors in Japanese patients with colorectal cancer: the significance of large bowel obstruction-univariate and multivariate analyses. J Surg Oncol 1991; 47: | 88-92

29. Muller S, Chesner IM, Egan MJ, Rowlands DC, Collard MJ, Swarbrick ET, et al. Significance of venous and lymphatic invasion in malignant polyps of the colon and rectum. Gut 1989; 30:1385-9.

30. Shanthaveerappa TR, Bourne GH. Perineural epithelium: a new concept of its role in the integrity of the peripheral nervous system. Science 1966; 154: | 464-7.

Artigo recebido: 23/12/02 Aceito para publicação: 22/04/03 